\title{
Video Article \\ A Micro-agar Salt Bridge Electrode for Analyzing the Proton Turnover Rate of Recombinant Membrane Proteins
}

\author{
Jürgen Kreiter ${ }^{1}$, Elena E. Pohl ${ }^{1}$ \\ ${ }^{1}$ Department of Physiology and Biophysics, University of Veterinary Medicine \\ Correspondence to: Elena E. Pohl at Elena.Pohl@vetmeduni.ac.at
}

URL: https://www.jove.com/video/58552

DOI: doi:10.3791/58552

Keywords: Neuroscience, Issue 143, Electrophysiology, electrogenic transport, diffusion potential, protein turnover number, Nernst potential, uncoupling proteins

Date Published: $1 / 7 / 2019$

Citation: Kreiter, J., Pohl, E.E. A Micro-agar Salt Bridge Electrode for Analyzing the Proton Turnover Rate of Recombinant Membrane Proteins. J. Vis. Exp. (143), e58552, doi:10.3791/58552 (2019).

\section{Abstract}

To date, more than $50 \%$ of all pharmacological drugs target the transport kinetics of membrane proteins. The electrophysiological characterization of membrane carrier proteins reconstituted in lipid bilayer membranes is a powerful but delicate method for the assessment of their physicochemical and pharmacological properties. The substrate turnover number is a unique parameter that allows the comparison of the activity of different membrane proteins. In an electrogenic transport, the gradient of the translocated substrate creates a membrane potential that directly correlates to the substrate turnover rate of the protein. By using silver chloride electrodes, a diffusion potential, also called liquid junction potential, is induced, which alters electrode potential and significantly disturbs precise membrane potential measurements. Diffusion potential can be minimized by a salt bridge, which balances electrode potential. In this article, a micro-agar salt bridge is designed to improve the electrophysiological set-up, which uses micropipettes for the membrane formation. The salt solution is filled into a microcapillary pipette tip, stabilized by the addition of agarose, and can be easily mounted to a standard electrode. The electrode potential of a micro-salt bridge electrode is more stable compared to a standard electrode. The implementation of this system stabilizes electrode potential and allows more precise measurements of membrane potential generated by a $\mathrm{pH}$ gradient. Using this system, the proton turnover rates of the mitochondrial carriers UCP1 and UCP3 are reinvestigated and compared to earlier measurements.

\section{Video Link}

The video component of this article can be found at https://www.jove.com/video/58552/

\section{Introduction}

Membrane proteins are targeted by up to $60 \%$ of all known pharmaceutical drugs ${ }^{1}$. Electrophysiological measurements of membrane proteins are a powerful but delicate tool to analyze the electrogenic transport of substrates mediated by membrane carrier proteins. The modulation of the transmembrane current by the application of constant voltages or voltage ramps allows assessing the pharmacological and physical properties of the carriers, for instance, the activation and inhibition by substrates or the transport kinetics. Of special interest is the substrate turnover number, which displays the amount of substrate that is translocated by a membrane protein per time unit. It is a major parameter when comparing the kinetics of various membrane proteins. Establishing a concentration gradient of the charged substrate across the membrane generates an electromotive force from which the turnover number of the substrate is deduced.

Using an $\mathrm{AgCl}$ electrode, the presence of a chloride-free buffer creates a diffusion potential that alters electrode potential and leads to a shift in current-voltage measurements ${ }^{2}$. Although always present, it is negligible for standard conductance and capacity measurements since these parameters are either dependent on the slope of the current-voltage recording (conductance) or are the difference of a single recording (capacity), which cancels the potential. However, the recording of the reverse potential, which is created by the transport of substrate, can be significantly disturbed by the diffusion potential. Thus, for exact measurements of the reverse potential, the electrode potentials have to be kept constant.

The diffusion potential can be minimized by two methods: (i) in the presence of a bilayer membrane, a substrate concentration has to be increased on one side of the membrane $e^{3,4}$, or (ii) a salt bridge balances the electrode potential ${ }^{5}$. The first method is highly dependent on the stability of the measurements. The membrane has to survive for several minutes, from the addition of substrate under stirring until the substrate is almost equally distributed in the solution. If the membrane ruptures in between, the substrate gradient is altered by the free exchange of charged molecules, and measurements turn inaccurate. The latter method balances the diffusion potential but is limited by the size of the set-up. Implementing a small but functioning salt bridge in a micro range to an electrophysiological set-up is challenging ${ }^{6}$. For the latter method, the salt solution is filled into a microcapillary tip and stabilized by the addition of agarose to prevent diffusion of the salt solution to the buffer solution.

In this protocol, a straightforward production of a micro-agar salt bridge and implementation into an electrophysiological set-up based on the pipette set-up ${ }^{7}$ is described. A microcapillary tip is adjusted both to contain a $3 \mathrm{M} \mathrm{KCl}$ solution with $1 \mathrm{~mol} \%$ (w/v) agarose and to bridge an $\mathrm{AgCl}$ electrode and buffer solution. The advantage of the micro-salt bridge is displayed by time recordings of the electrode potential shift and the more 
precise measurements of membrane potential at various $\mathrm{pH}$ gradients. In the model system of recombinant proteins reconstituted into liposomes, the turnover rates of mitochondrial carriers UCP1 and UCP3 produced under similar conditions are reinvestigated and compared to previous results ${ }^{3,8}$.

\section{Protocol}

\section{Production of Recombinant Uncoupling Proteins (UCPs) and the Formation of Planar Bilayer Membranes}

1. Produce recombinant UCP1 and UCP3 as described by Rupprecht et al. ${ }^{9}$ and Hilse et al. ${ }^{10}$

2. Form planar bilayer membranes on the tips of conventional dispensable plastic pipettes as described by Beck et al. ${ }^{7}$

\section{Preparation of the Micro-agar Salt Bridge Electrode}

1. Adjust a microcapillary pipette tip (see Table of Materials) to the appropriate length.

1. Mark the position on an empty tip at which the buffer-containing tip will be attached to and use a sliding caliper to measure the length of the micro-agar salt bridge electrode.

CAUTION: The microcapillary tip must be long enough to enter the buffer solution for the measurements.

2. Cut the microcapillary tip with a sharp knife or blade and clean the cut surface with ethanol and water.

2. Prepare the AgCl-coated electrode.

1. Cut off an $\mathrm{Ag}$ wire of approximately $8 \mathrm{~cm}$ in length and clean it with ethanol and water.

2. Take a piece of sandpaper and smooth down the surface of $1 \mathrm{~cm}$ length at the end of the wire, which should be in contact with the salt solution.

3. Dip the smoothed end into a $3 \mathrm{M} \mathrm{KCl}$ solution and coat it electrochemically with chloride by using a DC supply at $1.5 \mathrm{~V}$ for $10 \mathrm{~s}$.

4. Clean the electrode with water, dry it, and adjust the length of the $\mathrm{AgCl}$ electrode from the uncoated side so that it penetrates the microcapillary tip as deep as possible. Note: The protocol can be paused here.

3. Prepare a $3 \mathrm{M} \mathrm{KCl}$ salt solution with $1 \%(\mathrm{v} / \mathrm{v})$ agarose

1. Weigh out $4.47 \mathrm{~g}$ of $\mathrm{KCl}$ and dissolve it in $20 \mathrm{~mL}$ of water by stirring it in a flask.

2. Remove the stirrer, weigh out $0.2 \mathrm{~g}$ of agarose, and add it to the flask.

3. Heat up the solution to $100{ }^{\circ} \mathrm{C}$ to melt the agarose and prevent it from clotting. CAUTION: The flask will be very hot. Do not touch it with bare hands. Use gloves for handling.

4. Fill the microcapillary tip with the agarose salt solution.

CAUTION: The solution is hot. Protect hands and work carefully to avoid splashes.

1. If the agarose starts clotting, heat up the salt solution to fully melt the agarose again.

2. Soak $10 \mu \mathrm{L}$ of the agar salt solution into the microcapillary tip. Soak it slowly and carefully to avoid air bubbles in the tip.

3. Remove the tip from the pipette and push in the $\mathrm{AgCl}$ electrode from the broader side of the tip. Ensure that the electrode penetrates the salt solution.

4. Cool down the electrode to room temperature and plug it into the amplifier.

5. Prepare the buffer for the measurements.

1. Weigh out $0.710 \mathrm{~g}$ of $\mathrm{Na}_{2} \mathrm{SO}_{4}, 0.195 \mathrm{~g}$ of MES, $0.121 \mathrm{~g}$ of TRIS, and $0.023 \mathrm{~g}$ of EGTA, then add $100 \mathrm{~mL}$ of distilled water to a beaker and stir the solution.

2. Check for the $\mathrm{pH}$ value of the buffer by using a $\mathrm{pH}$ electrode and adjust the $\mathrm{pH}$ value to 7.32

3. Check that the reference electrode and the agar salt bridge electrode are in electrical contact.

1. Add $1 \mathrm{~mL}$ of buffer to a plastic container.

2. Dip in the reference electrode and the agar salt bridge electrode and check for a signal response. If the signal response is correct, proceed to step 2.7 .

6. If there is a false signal response or no electrical contact at all, perform the following troubleshooting.

1. Check if the electrode is in contact with the salt solution and push the electrode into the solution.

Note: If the solution is already too sticky, remove the micro-agar salt bridge and prepare a new microcapillary tip.

2. Check if there are air bubbles within the salt solution. If yes, prepare a new microcapillary tip.

3. Check if the salt solution is in contact with the buffer solution. If not, then cut off another $1 \mathrm{~mm}$ of the tube end of the tip. CAUTION: Be sure that the tip is still long enough to penetrate the buffer-containing tip. If there is still no contact, prepare a new microcapillary tip.

4. If none of these steps help, prepare a new microcapillary tip.

7. For storage, dip the agar salt bridge electrode into a $3 \mathrm{M} \mathrm{KCl}$ solution.

Note: The protocol can be stopped here. For a pause overnight, store the electrode in $3 \mathrm{M} \mathrm{KCl}$ salt solution at $4{ }^{\circ} \mathrm{C}$.

8. Prepare the buffer-containing plastic tip.

Note: If the electrode was stored overnight, take it out and let it heat up to room temperature for $30 \mathrm{~min}$.

1. Take a microcapillary tip and bend the tube, $2 \mathrm{~cm}$ from the edge of the narrow part, approximately 90 degrees using a heating wire.

2. Use a very sharp knife or blade and cut off the tube around $5 \mathrm{~mm}$ from the bend. 
3. Clean the surface at the end with ethanol and water and measure the diameter of the hole of the tip. From this, calculate the area of the surface.

4. Coat the surface of the tip with $85: 15$ (v:v) hexane:hexadecane.

1. Pipette $3 \mu \mathrm{L}$ of the solvent and remove it from the tip.

2. Wait 1 min so that all of the residual solvent in the tip has evaporated.

5. Fill the measurement tip with $3 \mu \mathrm{L}$ of buffer and plug it onto the salt bridge electrode.

NOTE: Check again if the salt bridge electrode and the reference electrode are in electrical contact by performing step 2.5.3.

6. If there is no electrical contact, check for the following:

1. Check whether the salt bridge electrode is in contact with the buffer solution. If not, then either increase the volume of the buffer in the tip or prepare a longer microcapillary tip.

2. If there is an air bubble, remove the tip from the micro-salt bridge electrode, refill the buffer in the tip, and plug it into the electrode again.

\section{Measurement of the Electrical Parameters of the Membrane Reconstituted with Recombinant Protein}

1. Apply a triangular alternating voltage signal with maximal voltage $U_{\max }=50 \mathrm{mV}$ and $\Delta T_{\text {ramp }}=50 \mathrm{~ms}$, which creates a rectangular alternating current response. From the mean values of the positive and negative currents $\left(\mathrm{I}_{+}\right.$and $\left.\mathrm{I}_{-}\right)$, calculate the capacity of the membrane according to the following formula:

$$
\mathrm{C}=\frac{\left(\mathrm{I}_{+}-\mathrm{I}_{-}\right) \cdot \Delta \mathrm{T}_{\mathrm{ramp}}}{2 \cdot \mathrm{U}_{\max }}
$$

2. Apply a voltage ramp ranging from $-50 \mathrm{mV}$ to $+50 \mathrm{mV}$ and record the current. Fit a linear function to the data - the slope is the conductance and calculate the $x$-axis intersection point of the fit.

3. Discharge the solution in the plastic tip and fill a new one with a buffer containing an increased substrate concentration.

1. If no membrane is formed within the first $20-30 \mathrm{~s}$, discharge the volume and refill it. This guarantees that the concentration gradient across the membrane does not significantly change during the membrane formation.

2. After the formation of a membrane, perform steps 3.1 and 3.2 again to verify proper membrane formation and to get the $x$-axis intersection point.

\section{Calculation of the Substrate Turnover Rate}

NOTE: See previous work for details ${ }^{3,7}$.

1. Estimate the amount of protein in the membrane from the molecular mass of lipid and protein ( $M_{\text {Lipid }}$ and $\left.M_{\text {Protein }}\right)$, the area of the membrane and of one lipid head group $\left(A_{\text {Membrane }}\right.$ and $\left.A_{L i p i d}\right)$, and the mass ratio of the protein per lipid $(r)$.

$$
\mathrm{N}_{\text {Protein }}=\frac{\mathrm{M}_{\text {Lipid }} \cdot \mathrm{A}_{\text {Membrane }}}{\mathrm{M}_{\text {Protein }} \cdot \mathrm{A}_{\text {Lipid }}} \cdot \mathrm{r}
$$

2. Calculate the Nernst potential for the transported substrate. $R$ is the gas constant, $T$ the temperature, $z$ the charge of the transported substrate, $F$ the Faraday's constant, and $c_{1}$ and $c_{2}$ the concentrations of the substrate of both sides of the membrane.

$\Phi_{\text {Nernst }}=\frac{\mathrm{RT}}{\mathrm{zF}} \cdot \ln \left(\frac{\mathrm{c}_{1}}{\mathrm{c}_{2}}\right)$

3. From the current-voltage recordings, take the reverse potential calculated with the difference of the $x$-axis intersection points from the linear fits in the presence and absence of the substrate gradient.

4. Calculate the proportion of substrate conductance, $\mathrm{G}_{\text {Substrate, }}$, to the total membrane conductance, $\mathrm{G}_{\text {total }}$, by the ratio of reverse potential to Nernst potential ${ }^{11}$.

$\mathrm{G}_{\text {Substrate }}=\frac{\Phi_{\text {reverse }}}{\Phi_{\text {Nernst }}} \cdot G_{\text {total }}$

5. Calculate the substrate turnover number $\Delta \mathrm{N}_{\text {Substrate }}$ per time unit $\Delta \mathrm{T}$ from the substrate conductance $\left(\mathrm{G}_{\text {Substrate }}\right)$, the applied voltage $\mathrm{U}$, and the charge of the substrate $\mathrm{z}$ :

$$
\frac{\Delta \mathrm{N}_{\text {Substrate }}}{\Delta \mathrm{T}}=\frac{\mathrm{G}_{\text {Substrate }} \cdot \mathrm{U}}{\mathrm{Z} \cdot \mathrm{e}}
$$

6. From the ratio of transported substrate per time to the number of proteins, calculate the turnover rate $\mathrm{k}$.

$$
\kappa=\frac{\Delta \mathrm{N}_{\text {Substrate }} / \Delta \mathrm{T}}{\mathrm{N}_{\text {Protein }}}
$$




\section{Representative Results}

To verify the minimization of the diffusion potential, the stability of the current-voltage measurements of an intact membrane was measured. In Figure 2A, representative-current voltage recordings are depicted in the presence (white dots) and absence (black dots) of a pH gradient. According to the Nernst equation, the $\mathrm{pH}$ gradient induces a shift in voltage. From the $\mathrm{x}$-axis intersection point of a linear fit to the data, the potential shift is calculated. In order to test both electrodes, the shift in $\mathrm{x}$-axis intersection point was analyzed for a standard $\mathrm{AgCl}$ electrode (Figure 2B; white dots) and a micro-agar salt bridge (Figure 2B; black dots). A voltage ramp was recorded ten times in a row and the mean shift in the $\mathrm{x}$-axis is depicted against time. Whereas the agar salt bridge electrode had a maximum shift of less than $5 \mathrm{mV}$ even after 300 seconds of measurement, the standard electrode varied up to $30 \mathrm{mV}$ in unpredictable, random, behavior.

Next, both electrodes were tested at different $\mathrm{pH}$ gradients (Figure 3A). For the standard electrode, a pH gradient of 0.35 and 1.0 (white dots) was generated; for the agar salt bridge electrode, $\mathrm{pH}$ gradients of $0.35,0.7$, and 1.0 (black dots). The shift in potential was analyzed in three independent measurements. In contrast to a gradient of 0.35 , where the measured shift only varies slightly, the voltage shift significantly alters at the $\mathrm{pH}$ gradient of 1.0 in the absence of the micro-agar salt bridge. From a linear fit to the data, the slope of the function is $26.4 \pm 2.3 \mathrm{mV} / \Delta \mathrm{pH}$ for the standard electrode and $50.1 \pm 4.6 \mathrm{mV} / \Delta \mathrm{pH}$ for the micro-agar salt bridge electrode. According to the Nernst equation, the calculated potential shift is $60.7 \mathrm{mV} / \Delta \mathrm{pH}$ at $\mathrm{T}=32{ }^{\circ} \mathrm{C}$.

Using the micro-agar salt bridge, the proton turnover number, $\mathrm{K}$, of mitochondrial UCP1 and UCP3 was measured and compared to previous measurements (Figure 3B). Similar to Figure $3 \mathbf{A}, \Delta \mathrm{pH}=1.0$ was generated and the reverse potential was measured. The amount of protein in the membrane was estimated according to the formula provided in section 4 of the protocol, with a protein to lipid ratio of $4 \mu \mathrm{g} /(\mathrm{mg}$ of lipid), a molecular mass of 33,000 , and $750 \mathrm{Da}$ for the protein and lipid, a membrane surface area of $3.53 \times 10^{-4} \mathrm{~cm}^{2}$, and an area per lipid of $7.8 \times 10^{-15}$ $\mathrm{cm}^{2}$. The obtained kwas $5.56 \pm 0.38 \mathrm{~s}^{-1}$ and $4.10 \pm 0.71 \mathrm{~s}^{-1}$ for UCP1 and UCP3, respectively (Figure 3B).

A

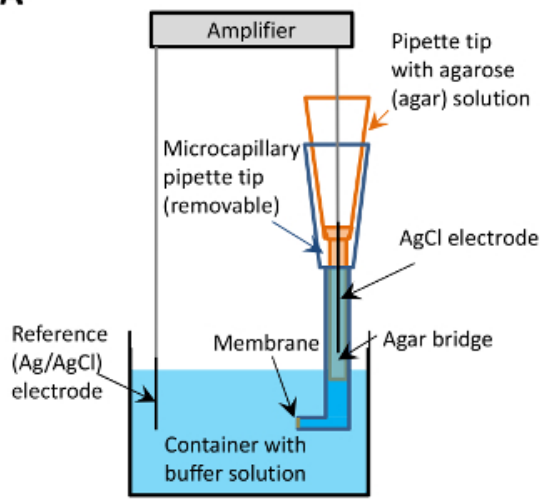

B

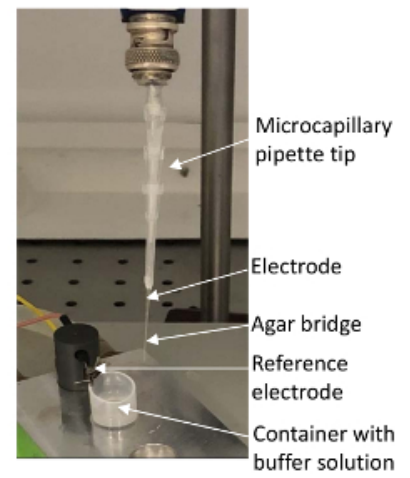

Figure 1: The electrophysiological set-up with the micro-agar salt bridge. (A) This panel shows a sketch of the set-up. The microcapillary tip containing the agar salt solution (depicted in orange) is placed between the electrode (black) and the buffer-containing tip (blue). The membrane is formed at the surface at the end of the buffer-containing tip (indicated by the arrow). (B) This panel shows an image of the electrophysiological set-up with the implementation of the micro-agar salt bridge. The arrows point to the electrode, the micro-agar salt bridge, the reference electrode, and the container with buffer solution. Please click here to view a larger version of this figure. 
A

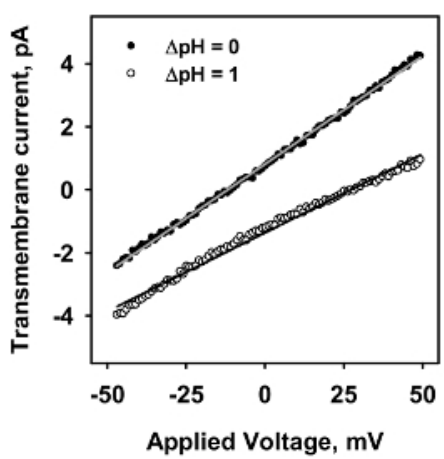

B

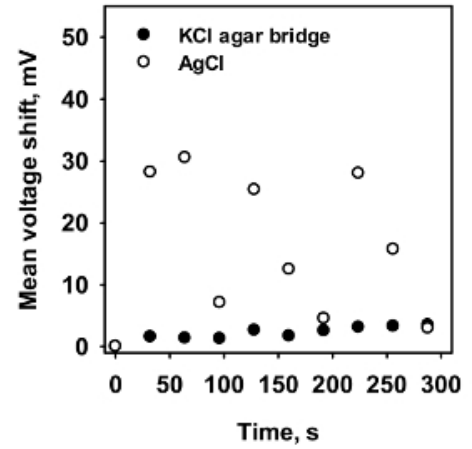

Figure 2: The comparison of a micro-agar salt bridge and a standard $\mathrm{AgCl}$ electrode. (A) This panel shows a representative currentvoltage measurement in the presence (grey dots) and absence (white dots) of a pH gradient of 1 . The lines represent a linear fit to the data, from which conductance and $\mathrm{x}$-axis intersection values are obtained. The voltage shift is evaluated by the difference of the intersection values of both recordings. (B) This panel shows the shift of membrane potential of a standard $\mathrm{AgCl}$ electrode (white dots) to a micro-agar salt bridge electrode (black dots) in time. Ten current-voltage measurements were recorded in a row and the mean voltage shift by diffusion potential is plotted against time. In all experiments, the membrane was made of 45:45:10 mol\% DOPC:DOPE:CL reconstituted with 15 mol\% arachidonic acid at a concentration of $1.5 \mathrm{mg} / \mathrm{mL}$. The buffer contained $50 \mathrm{mM} \mathrm{Na}_{2} \mathrm{SO}_{4}, 10 \mathrm{mM} \mathrm{MES}, 10 \mathrm{mM} \mathrm{TRIS}$, and $0.6 \mathrm{mM} \mathrm{EGTA}$ at pH = 7.34 and T = $32{ }^{\circ} \mathrm{C}$. Please click here to view a larger version of this figure.

A

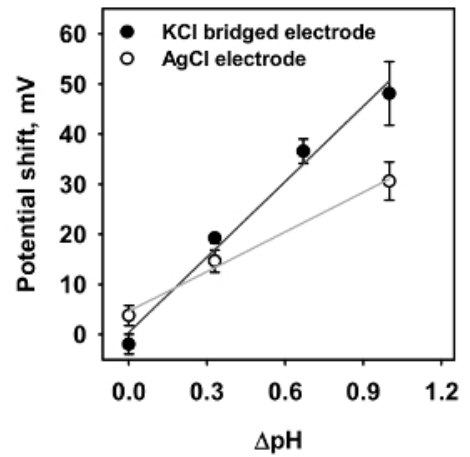

B

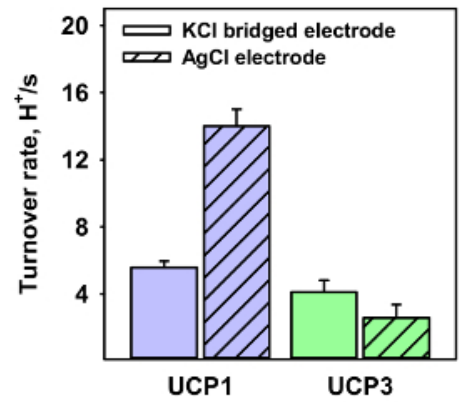

Figure 3: Proton turnover number of UCP1 and UCP3 calculated from the reverse potential in the presence of a pH gradient. (A) This panel shows the shift in potential of a UCP1-containing membrane of various $\mathrm{pH}$ gradients of a standard $\mathrm{AgCl}$ electrode (white dots) and a microagar salt bridge electrode (black dots). The lines represent a linear fit to the data. (B) This panel shows the proton turnover number of UCP1 (first data set) and UCP3 (second data set) as calculated from the ratio of voltage shift to Nernst potential according to the formulas in section 4 of the protocol. The first bar of each set represents turnover rates measured with the micro-agar salt bridge. The second bar of each data set represents previous measurements using a standard $\mathrm{AgCl}$ electrode. Values for UCP1 and UCP3 were taken from Urbankovaet al. ${ }^{3}$ and Macher et al. $^{8}$. In all measurements, the membrane was made of 45:45:10 mol\% DOPC:DOPE:CL reconstituted with 15 mol\% AA and UCP1/UCP3. The concentration of lipid and protein was $1.5 \mathrm{mg} / \mathrm{mL}$ and $4 \mu \mathrm{g} / \mathrm{mg}$ of lipid, respectively. The buffer contained $50 \mathrm{mM} \mathrm{Na}_{2} \mathrm{SO}_{4}, 10 \mathrm{mM} \mathrm{MES}, 10$ $\mathrm{mM}$ TRIS, and $0.6 \mathrm{mM}$ EGTA at $\mathrm{pH}=7.34$ and $\mathrm{T}=32^{\circ} \mathrm{C}$. The $\mathrm{pH}$ of the buffer for the gradient measurements was increased to $7.66,8.00$, or 8.33 by adding TRIS and was changed in the solution-containing pipette. The values are the mean \pm standard deviation of three independent measurements. Please click here to view a larger version of this figure.

\section{Discussion}

The implementation of the micro-agar salt bridge with the electrode minimizes its diffusion potential and allows more precise measurements of membrane potential generated by a $\mathrm{pH}$ gradient. In the presence of various transmembrane $\mathrm{pH}$ gradients, the potential shift of both electrodes was acceptable at $\Delta \mathrm{pH}=0.35$ when comparing to the theoretical value of the Nernst equilibrium potential $\left(\Phi_{\text {Nernst }}=23.8 \mathrm{mV}\right.$ for $\left.\Delta \mathrm{pH}=0.4\right)$. However, at more physiological $\mathrm{pH}$ gradients, as for instance in mitochondria between the matrix and intermembrane space, the standard $\mathrm{AgCl}$ electrode failed to precisely measure the potential shift at $\Delta \mathrm{pH}=1$ (Figure 3A). The electrode bridged with micro-agar salt delivered the values which were much more comparable to the theory.

Diffusion potential may also occur at the $\mathrm{AgCl}$ reference electrode if the buffer solution is changed during the experiment. Chloride-free buffer solution was used in the experiments since uncoupling proteins were suggested to transport chloride ions, and the $\mathrm{pH}$ was adjusted using Tris or MES. The electrode potential, in the absence of a substantial concentration of chloride, primarily depends on chloride impurities in the buffer solution. As its composition is unchanged during the experiments, it will simply result in a constant offset potential. However, for the measurement of an absolute potential difference between the two electrodes, a simple agar salt-bridge system $(\mathrm{Ag} / \mathrm{AgCl} 3 \mathrm{M} \mathrm{KCl}) \mathrm{could}$ also be used for the reference electrode. 
A micro-agar salt bridge balances the diffusion potential by an equilibration of the electrode potential. In order to stabilize the salt solution, $1 \%$ $(\mathrm{w} / \mathrm{v})$ agarose was added to prevent the mixing of the salt solution with the buffer solution. The salt ions $\mathrm{K}^{+}$and $\mathrm{Cl}^{-}$have similar mobilities in liquid and balance the electrode potential. To properly install the salt bridge, the agar salt solution has to be sufficiently heated up to fill the microcapillary tip without any air bubbles and to cover the $\mathrm{AgCl}$ electrode. Before further usage, electrical contact between the salt bridge electrode and the reference electrode has to be checked. Depending on the time the salt bridge is used, the salt solution has to be sufficiently gelled to prevent any mixing of the salt solution with the buffer. This is especially critical if $\mathrm{K}^{+}$or $\mathrm{Cl}^{-}$transporters are investigated. The salt bridge was used for a very short time and the elution of agarose is negligible in this time range. A higher concentration of agarose of up to $5 \%$, or of agar $(3 \%-5 \%)$, allows using the salt bridge for a longer period of time ${ }^{6,12}$.

This method allows determining the transport kinetics of a membrane transporter (i) with low turnover rates and (ii) of mitochondrial proteins of the inner membrane, which can hardly be investigated in standard patch clamp set-ups ${ }^{13}$. Its precision is mainly dependent on the reverse potential measurement, which accuracy is decreased at a low total membrane conductance and small concentration gradients which induce a membrane potential below the noise of recording.

Using this set-up, the turnover rates of UCP1 and UCP3 as produced under the same conditions were measured. Due to the higher pH gradient, the obtained rates seem to be more precise and unperturbed by artefacts resulting from the minor electrode potential shift. It can be used to further analyze and compare mitochondrial membrane transporters produced under similar conditions.

\section{Disclosures}

The authors have nothing to disclose.

\section{Acknowledgements}

This work was supported by the Austrian Research Fund (P31559-B20 to E.E.P.). The authors thank Sarah Bardakji for the excellent technical assistance in the production and reconstitution of mouse UCP1 and UCP3 into proteoliposomes.

\section{References}

1. Terstappen, G. C., Reggiani, A. In silico research in drug discovery. Trends in Pharmacological Sciences. 22 (1), $23-26$ (2001).

2. Raynauld, J. P., Laviolette, J. R. The silver-silver chloride electrode: A possible generator of offset voltages and currents. Journal of Neuroscience Methods. 19 (3), 249-255 (1987).

3. Urbankova, E., Voltchenko, A., Pohl, P., Jezek, P., Pohl, E. E. Transport kinetics of uncoupling proteins. Analysis of UCP1 reconstituted in planar lipid bilayers. Journal of Biological Chemistry. 278 (35), 32497-32500 (2003).

4. Beck, V. et al. Polyunsaturated fatty acids activate human uncoupling proteins 1 and 2 in planar lipid bilayers. The FASEB Journal. 21 (4), 1137-1144 (2007).

5. Shao, X. M., Feldman, J. L. Micro-agar salt bridge in patch-clamp electrode holder stabilizes electrode potentials. Journal of Neuroscience Methods. 159 (1), 108-115 (2007).

6. Kleene, S. J. A simple intrapipette salt bridge. Journal of Neuroscience Methods. 46 (1), 11-16 (1993).

7. Beck, V. et al. A new automated technique for the reconstitution of hydrophobic proteins into planar bilayer membranes. Studies of human recombinant uncoupling protein 1. Biochimica et Biophysica Acta-Bioenergetics. 1757 (5-6), 474-479 (2006).

8. Macher, G. et al. Inhibition of mitochondrial UCP1 and UCP3 by purine nucleotides and phosphate. Biochimica et Biophysica Acta. 1860 (3), 664-672 (2018).

9. Rupprecht, A. et al. Role of the transmembrane potential in the membrane proton leak. Biophysical Journal. 98 (8), $1503-1511$ (2010).

10. Hilse, K. E. et al. The expression of UCP3 directly correlates to UCP1 abundance in brown adipose tissue. Biochimica et Biophysica Acta. 1857 (1), $72-78$ (2016).

11. Fuks, B., Homble, F. Permeability and electrical properties of planar lipid membranes from thylakoid lipids. Biophysical Journal. 66 (5), 1404-1414 (1994).

12. Barry, P. H., Lewis, T. M., Moorhouse, A. J. An optimised $3 \mathrm{M} \mathrm{KCl}$ salt-bridge technique used to measure and validate theoretical liquid junction potential values in patch-clamping and electrophysiology. European Biophysics Journal. 42 (8), 631-646 (2013).

13. Huang, D., Li, J. The feasibility and limitation of patch-clamp recordings from neonatal rat cardiac ventricular slices. In Vitro Cellular \& Developmental Biology. 47 (4), 269-272 (2011). 